\title{
La recepción de la noción procleana de authypóstaton en Nicolás de Cusa
}

\author{
CLAUDIA D’AMICO \\ Consejo Nacional de Investigaciones Científicas y Técnicas \\ Universidad de Buenos Aires \\ Universidad Nacional de La Plata
}

DOI: $10.36446 /$ rlf2021211

Resumen: Este artículo analiza la noción de authypóstaton en la obra De principio (1459) de Nicolás de Cusa a partir de la lectura directa que realiza el autor de dos escritos procleanos en su versión latina, Elementatio Theologica e In Parmenidem. Tal noción es desplazada del ámbito de las hénadas a la del principio primero en una doble consideración, como principio intelectual y como principio supraintelectual. Sin embargo, procura mostrar que la apropiación de esta noción que realiza el Cusano supone una tradición de lectura en la cual él se inscribe.

Palabras clave: Proclo latino, autoconstitución, principio intelectual, principio supraintelectual. 


\title{
The Reception of the Proclean Notion authypóstaton in Nicholas of Cusa
}

\begin{abstract}
In this paper I analize the notion of authypóstaton in Nicolás of Cusa's treatise De Principio (1459). I will specifically explore Cusanus' interpretation of this concept based on his direct access to Proclus' Elementatio Theologica and In Parmenidem, i.e., two Proclean works in their Latin version. I will claim that Nicholas displaced the notion of authypostaton from the domain of henads to that of the first principle. Thus, it becomes both an intellectual and a supra-intellectual principle. However, the assimilation of this notion by Cusanus shows that he was consciously enrolled in that philosophical tradition.
\end{abstract}

Key-words: Latin Proclus, self-constitution, intellectual principle, supra-intellectual principle.

Ta recepción de Proclo en la Edad Media latina está siendo estudiada como nunca antes. A los fundacionales estudios de Klibansky (1929), R. Imbach (1978), L. Sturlese (1987) junto con las ediciones de Steel (Proclus 1982-1985) y Böese (Proclus 1987)1 , le siguieron investigaciones del propio Steel $(2014)$, S. Gersh $(2014,2017)$ y D. Calma (2019) quien dirige actualmente un gran proyecto de investigación que tiene al menos dos volúmenes más en preparación. La lista no pretende ser exhaustiva y se completa con innumerables trabajos sobre autores medievales y su particular recepción de la obra procleana.

Es habitual poner de manifiesto distintas etapas en la recepción del Proclo latino. Sin tener en cuenta como relevante una temprana traducción de autor desconocido de Elementatio Physicae hacia 1160 (Imbach 1978: 431), los estudios se concentran en una primera gran etapa de recepción atravesada por la fascinación que produjo sobre todo en la Universidad de París la Elementatio Theologica, cuya versión al latín fue culminada por Moerbeke en 1268. Su construcción geométrica causó un gran efecto en un contexto en el cual uno de los problemas fundamentales era el carácter "científico" de la teología (Sturlese 1987:262). Además de la Elementatio, Moerbeke realizó sus versiones de Tria opuscula (1280), una parte de In Timaeum y la totalidad de In

\footnotetext{
${ }^{1}$ Las remisiones a las obras y las citas textuales del Proclo latino seguirán estas ediciones citadas de manera completa en la lista bibliográfica que se pospone.
} 
Parmenidem (1286). Hacia fines del siglo XIII, cuando los textos procleanos comienzan a perder interés en París, lo ganan en Alemania entre autores con intereses diversos, desde un enciclopedista como Enrique Bate de Malines a un maestro universitario como Dietrich de Freiberg. En los primeros decenios del siglo XIV la revalorización de Proclo en tierras germanas ya no está conducida por el deseo de encontrar un fundamento racional para la teología sino más bien por el de encontrar las bases que permitan romper los límites entre la filosofía y la teología, además de entre las diversas religiones y escuelas filosóficas. Como claramente puede leerse en el gran comentario de Bertoldo de Moosburg a la Elementatio Theologica, Proclo se considera el portavoz de una verdadera y propia revelación filosófica deudora de una sapientia antiquísima (Sturlese 1987: 262-263). De esta última recepción germana, un siglo más tarde, es deudor, en gran medida, el pensamiento de Nicolás de Cusa.

Es bien sabido y ha sido estudiada suficientemente la lectura directa que hace el Cusano de los textos de Proclo (Klibansky 1929; Beierwaltes 1987, Senger 1986; Flasch 1998; D’Amico 2009, 2011; Gersh 2014; Secchi 2018). Algunas obras de Proclo se encuentran en su propia biblioteca conservada hasta hoy en Bernkastel-Kues. Los códices son: Procli De theologia Platonis Libri VI (CC 185), Procli Expositio in Parmenidem Platonis (CC 186) y Procli Elementatio theologica (CC 195). Cabe señalar que se trata de las versiones latinas de estas obras: In Parmenidem y Elementatio en la traducción de Guillermo de Moerbeke y De Platonis Theologia en la versión completa que realizó en su propio tiempo su amigo Pedro Balbo. Todas las obras de Proclo presentan notas marginales que han sido editadas (Senger,1986; Bormann 1986). Sin embargo, así como no todas las obras fueron recibidas ni asimiladas al mismo tiempo, tampoco fueron anotadas de una sola vez. Los datos de recepción de las obras por parte de Nicolás de Cusa pueden aportar una señal, a excepción del ejemplar de la Elementatio theologica, de la cual no se sabe con precisión acerca de su adquisición (Senger 1986:17-18).

Si bien esta lectura directa es decisiva, el tema de este artículo, la noción de authypóstaton y sus implicaciones, no puede ser abordada sin considerar otros pensadores anteriores al Cusano que tomaron posición frente a este tópico y cuyas obras el Cusano conoce bien. Es importante, pues, tenerlos en cuenta como una suerte de mediación para la lectura directa, una especie de lente que permite resolver algunas cuestiones de manera conveniente.

La noción de authypóstaton o la autoconstitución es para Proclo fundamentalmente la característica propia de las hénadas o dioses y su tratamiento involucra para un pensador cristiano la cuestión del politeísmo, en consecuencia la imposibilidad de concordancia entre el pensamiento de este 
filósofo ateniense y la sapientia nostra christiana. De este modo, si en algunas lecturas la intención del autor cristiano es el uso de Proclo para la búsqueda de acuerdos filosófico-teológicos la resolución de este tópico no es menor.

Veamos en primer lugar cómo se presenta el tema en los escritos procleanos conocidos por el Cusano. Como ha afirmado Gersh, la doctrina de los authypóstata o de los seres autoconstituidos es una de las más interesantes de la metafisica de Proclo; sin embargo, se vuelve problemática al tratarla como si obedeciera a una lógica estática. La autoconstitución es parte del movimiento del mundo espiritual procleano -atemporal e inespacial- y en este sentido está sometida a una lógica dinámica. Los seres que se constituyen a sí mismos son una parte fundamental de la cadena causal establecida por Proclo y forman parte de la explicación de la procesión desde lo uno a lo múltiple (Gersh 1973: 7-12).

Si bien se han señalado antecedentes de esta doctrina en Jámblico (Dodds 1963: XXI), lo cierto es que la Edad Media latina conoce los términos referidos a la autoconstitución solo a través de las obras de Proclo. De hecho, el término no se encuentra en el célebre Liber de Causis, texto en el cual no se incluye a las hénadas.

La presentación más sistemática aunque no exclusiva del tema se encuentra en Elementatio Theologica y ocupa un grupo de proposiciones desde 40 a 51, aunque hay antecedentes en las anteriores y se retoma en alguna de las posteriores. El objetivo de Proclo es diferenciar jerárquicamente los seres que proceden de una causa externa a la cual se subordinan, de aquellos que reciben de sí mismos su existencia y entonces se autoconstituyen ( $f f r$ Prop. 40). Este tema se vincula al que Proclo había presentado en proposiciones anteriores, el de la autarquía o autosuficiencia (autarkés). Es autárquico aquel que en sí mismo y desde sí mismo posee el bien, está pleno de bien. Pero aquello de lo que está pleno está por encima de él, por tanto el Bien absoluto no es autárquico. Esta autarquía (autárkeia) se da en dos planos: el del ser (ousía) y el de la actividad (enérgeia) (cfr. Prop.10).

Los authypóstata son también autárquicos, no existen "en otro" sino "en sí mismos" y por esto no tienen necesidad de un sustrato (hypokeímenon): proceden de sí mismos y se vuelven a sí mismos (cfr. Prop. 41). Precisamente, esta capacidad de volverse a sí mismos los hace autosuficientes y perfectos (autárkei kai téleion) (cfr. Prop. 42/43). El retorno se da en ambos aspectos: en cuanto a su ser y en cuanto a su actividad (cfr. Prop. 44). Así, todo authypóstaton es no generado (agéneton). Su ser se autoproduce eternamente ( $c f r$. Prop. 45). No está sujeto a corrupción en cuanto solo se corrompe lo que está separado de su causa. Estar unido a la causa es condición de integridad y conservación ( $f$ r. Prop. 46). Es simple y carece de partes ( $f$ r. Prop. 47). Sin embargo, su simplicidad es "relativa" en tanto está sujeta a la oposición divi- 
sible-indivisible / compuesto-simple. No está sujeto al tiempo, a la sucesión ni al número pues todo lo sujeto a sucesión tiene su ser en el no ser (cfr. Prop. 49/50) y por tanto está separado de lo que padece una medición temporal (cfr. Prop. 51).

La autoconstitución y autarquía son, pues, similares. Ambas remiten a la anterioridad respecto de otros seres: anterioridad respecto de aquellos que tienen una causa externa. Y así como los seres autárquicos reciben su plenitud de lo que está por sobre él, la anterioridad de los autoconstituidos tampoco es primera: los authypóstata son ellos mismos posteriores a lo Uno. En suma, para Proclo autarquía y authypóstasis no se predican del Principio primero.

Conviene revisar qué sucede con estos términos "técnicos" del vocabulario procleano en las versiones latinas de sus obras. Debemos señalar que Moerbeke elige mantener los términos en griego poniendo junto a ellos una locución latina equivalente y antecedida por un "id est". Así para "autarkés" elige como equivalente "per se sufficiens" (Prop. 10:"Quid enim aliud est autarkes (id est sibi sufficiens)..." у para "authypóstaton", "per se subsistens" (Prop. 40:"... Omnia que ab alia causa procedunt precedunt que a se ipsis subsistunt et habentia essentiam authypóstaton (id estper se subsistentem)"). Otros términos que aluden a otras características los traduce obviando el griego; esto sucede por ejemplo con los vocablos "ingenerabile" (agéneton) o "perpetuum" (aíon).

En latín ambas expresiones, "per se sufficiens" y "per se subsistens", son muy similares, ambas indican suficiencia ontológica. Sobre todo en la traducción del "authypóstaton" por el "per se subsistens" se pierde el movimiento de autoconstitución, es decir salir de sí para retornar a sí.

Nicolás de Cusa trata este tema en su opúsculo De Principio de 1459. En este escrito pone en relación de manera explícita su propio pensamiento con la filosofia de Proclo. La obra mencionada allí no es Elementatio Theologica -que ya había leído y anotado-sino In Parmenidem que había hecho copiar dos veces hacia 1450 para regalar un ejemplar a su amigo el Papa Nicolás V y otro ejemplar para su propio uso, en el cual trabajó profusamente, como lo muestran la multitud de notas que colocó en sus márgenes (Bormann 1986).

Las referencias que hace a esta obra procleana en De Principio corresponden sobre todo a los libros VI y VII y sus referencias son rastreables en la edición crítica de la obra y los estudios publicados por K. Bormann (2001, 2002). Si atendemos al texto procleano, es necesario indicar que el libro VII de In Parmenidem tiene la particularidad de que no se ha conservado en su totalidad en griego. La última parte solo se conserva en la versión de Moerbeke. Las menciones al authypóstaton se concentran entre Co 1145-1150, en el comentario a la primera hipótesis. Esta parte del texto en el manuscrito cusano tiene una gran cantidad de marginales de su propia mano. 
Proclo comenta a Platón. El Cusano, en la mayoría de los casos, repite las palabras de Proclo que opacan en gran medida la intención inicial del texto comentado. En esta sección, Proclo comenta aquel pasaje de Parménides donde Platón afirma que "lo uno no puede estar en ningún lugar puesto que no puede estar en otro (in alio) ni en sí mismo (in se ipso)". En primer lugar, se niega que esté "en otro". Proclo introduce entonces la noción de authypóstaton comentando un pasaje en el cual Platón afirma que si lo uno estuviera “en sí mismo" estaría rodeándose a sí mismo, puesto que está en sí mismo y es imposible estar en algo y no ser rodeado (continens) por él (138 A 7- B 2) Concluye Proclo, entonces: "Dicitur quidem igitur in se ipso esse omne quod sui ipsius causa et quod authypóstaton est".

Es en sí mismo lo que halla su causa en sí mismo, es decir lo que se autoconstituye. En este texto nuevamente el authypóstaton está vinculado con la causalidad. En lo que sigue, al comentar Parménides 138 B 2-5 (Co 11471151) Proclo concentra el vocabulario acerca del authypóstaton. Al revisar la versión latina advertimos que Moerbeke vuelve a elegir dejar el término en griego, pero esta vez sin aclaración en latín. En este tramo del comentario, al igual que en la Elementatio, cuando Proclo habla del authypóstaton no lo refiere en ningún caso al principio primero.

La insistencia en resaltar esto último reside en el hecho de que el Cusano en la obra De Principio retoma casi literalmente y mencionando el Comentario lo que Proclo dice allí del authypóstaton, sin embargo, a diferencia de Proclo, como veremos, refiere esta propiedad solo al principio primero. En lo que sigue intentaré mostrar que no se trata de una mala comprensión del texto de Proclo sino de una reapropiación del término que supone la consideración de otros actores.

En efecto, si revisamos las marginales al texto que se encuentran en el Codex Cusanus 186 advertimos que se repite casi literalmente el texto de Proclo. En la margen de Co 1150 hay una anotación que vale la pena subrayar porque el Cusano escribe -repitiendo el texto que lee- que lo uno mismo no es authypóstaton sino causa de los authypóstata. Leemos en la marginal: "unum non est autypostaton, quia ipsum qualitercumque divisibile, sed non unum; est enim unum, authypostatorum causa" (Bormann 1986: 134).

Baste este ejemplo para señalar que el Cusano ha leído y comprende lo que Proclo sostiene. Con todo, a pesar de que el acceso a la obra del diádoco de Platón es directo, ya hay una historia de la recepción de algunas nociones que el Cusano parece conocer bien. En esa historia, algunos autores cristianos han modificado el sentido de los authypóstata, eliminando o reconvirtiendo el plano de las hénadas o dioses en otro tipo de entidad o bien directamente eliminándolo. Con todo, podríamos preguntarnos por qué si se resignifica o elimina el plano de las hénadas o authypóstata, 
se mantiene la noción misma de authypóstaton y se la predica del primer principio.

Cualquier historia de la recepción de doctrinas procleanas en la Edad Media latina no puede obviar la referencia al Liber de Causis, sin embargo, como anticipamos, no aparece en él la noción de authypóstata. Con todo, se afirma en la Proposición XIV (XV) que todo cognoscente que conoce su esencia retorna a ella con una reditio completa. Esta suerte de autoconocimiento que es al mismo tiempo autoconstitución nunca se afirma de la primera causa. De los autores anteriores al Cusano que tuvieron una lectura directa de los escritos de Proclo traducidos por Moerbeke, es necesario señalar especialmente dos cuyas obras el Cusano conoció y que podemos decir que, en cierto sentido, anticipan su interpretación del authypóstaton. Se trata en ambos casos de autores procedentes de la línea germánica, uno Enrique Bate de Malines, el otro Bertoldo de Moosburg. Ambos escribieron sendas obras monumentales: la gran enciclopedia de Enrique titulada Speculum divinorum y el primer y extenso comentario a Proclo, la Expositio sobre la Elementatio Theologica de Bertoldo de Moosburg.

Sabemos que el Cusano conoció estas obras: ambas son mencionadas en su Apologia doctae ignorantiae. Por lo demás, se ha verificado que la obra de Bate está repleta de marginales de propia mano del Cusano en un códice de la Biblioteca Real de Bruselas (Codd. Bruxellensis 271) y se ha comparado una de esas marginales con la mención de la Apología relativa a la coincidencia entre la unidad y la pluralidad en las sustancias intelectuales (van de Vyver 1956: 442) El pasaje en el que el Cusano menciona a Bate en la Apología, escrito en el cual tuvo que defenderse de una acusación de heterodoxia, se encuentra inmediatamente después de aquel en el cual sostiene que Dios está más allá de la coincidencia de los opuestos. Esta doctrina que, recuerda, había sostenido en De coniecturis se refuerza en esta obra con una fórmula de Dionisio: Dios es la oposición a los opuestos. Enrique, quien se ubicaría también en la línea de continuidad que reúne a Proclo con el Areopagita, fue conducido, según el Cusano, a la contemplación de la coincidencia de unidad y pluralidad en las realidades intelectuales.

Fuit aliquando Henricus de Mechlinia, ut scribit in Speculo divinorum, ad hoc ductus, ut in intellectualibus conspiceret unitatis et pluralitatis coincidentiam, de qua plurimum admiratur (Apol., n. 22). ${ }^{2}$

\footnotetext{
${ }^{2}$ Las citas de las obras cusanas corresponden a la edición de la Academia de Heidelberg. En todos los casos nos limitaremos a colocar el número de parágrafo correspondiente a esa edición. Ver datos completos en la lista bibliográfica que se pospone.
} 
Enrique en el Speculum divinorum "corrige" la doctrina procleana del authypóstaton y el autarkés. Objeta a Proclo afirmando que solo puede ser llamada así, en sentido estricto, la primera causa. Pone en contradicción a Proclo consigo mismo confrontando las Proposiciones 10 y 127 (Steel 1990: LXII). En la Prop. 127 de la Elementatio se enuncia que todo aquello que es divino es simple a nivel primario y en el máximo grado: en consecuencia es absolutamente autosuficiente, en la versión de Moerbeke: "Omne divinum simplex prime est et maxime et propter hoc maxime per se sufficiens". Por otra parte, la interpretación del "per se subsistens" de Enrique se sustrae de la interpretación parisina en tanto no interpreta el "dei" en un sentido politeísta sino como virtudes derivadas o inteligencias celestes. En ese tratamiento la primera causa aparece mencionada como "intellectus" con apoyo en múltiples pasajes del libro XII de la Metafísica de Aristóteles a lo que se añade una nota típicamente procleana: la imparticipabilidad ( $f$ r. Speculum divinorum, XI 28, p. 101,1.37-43). Como ha sostenido recientemente Guildentops, esta reconciliación se configura por dos movimientos complementarios, a saber, una interpretación neoplatónica de Aristóteles y una simplificación de la metafísica procleana: el primer intelecto subsume en sí las henéadas y las ideas divinas así como la tríada ser, vida y pensamiento (Guildentops 2019:365-367).

Muchos de estos elementos señalados en la obra de Bate, si no todos, se encuentran en el planteo cusano en un marco general que también es el de Bate: la búsqueda de concordancia entre platonismo y aristotelismo, en el cual tanto para uno como para otro hay una clara preferencia por la tradición platónica.

Respecto de Bertoldo de Moosburg no podemos dejar de mencionar las relaciones entre su pensamiento y el Cusano señaladas por Flasch (1984: XXXV-XXXVIII) al introducir la primera edición crítica de la obra del dominico teutónico. El fundamento de que el Cusano conoce la Expositio se encuentra también en la Apología doctae ignorantiae donde el Cusano lo pone del lado de aquellos autores cuyos escritos deben ser sustraídos de los ojos de los que no pueden comprenderlos. La obra de Bertoldo es nombrada como Commentaria fratris Iohannis de Mossbach in Propositiones Proculi en una lista donde figuran además Dionisio, Mario Victorino, Honorius Augustodinensis, Escoto Eriúgena y David de Dinant (cfr. Apol. n. 43). La obra de Bertoldo, como la de Enrique y la de la totalidad de los escritos cusanos, transita el camino de la búsqueda de acuerdos entre distintas tradiciones filosóficas y teológicas. El apego al texto de Proclo que está comentando hace que ponga el principio primero o uno, en primera instancia, por encima del ser y del intelecto. Como ha mostrado Ludueña (2016:51), la noción de authypóstaton o per se subsistens es aplicada al nivel de las unitates o bonitates -hénadas procleanas- (cfr. Expositio 10A, vol. I, p. 177 y Expositio 43D, vol. III, p. 70), 
nivel que Bertoldo no suprime y reinterpreta a la luz de la doctrina eriugeniana de las "causas primordiales", solucionando de este modo el politeísmo procleno. Estas unitates se autoconstituyen y por eso pueden ser llamadas authypóstata; sin embargo, el término también puede predicarse de lo que no tiene un agente superior que lo cause y en este sentido se aplica también con propiedad a lo prime bonum et unum, el cual, también en algún sentido, por más que sea supraintelectual, es explicado como intelecto cuando debe atenderse a su constitución trina ( $f f r$. Ludueña en este mismo volumen). Esta tensión entre la consideración del principio primero como supraintelectual e intelectual, se repetirá en el Cusano.

Podemos decir como una suerte de conclusión provisoria que una línea de interpretación del pensamiento de Proclo no suprimió sino que antes bien privilegió la noción de autoconstitución pero la desplazó desde el territorio de las hénadas al del primer principio y utilizó esta noción como herramienta conceptual para esclarecer, de algún modo, la constitución unitrina del Dios cristiano. Complementariamente, esto explicaría también por qué la noción de autoconstitución aparece con otros términos en la redefinición del pensamiento procleano por parte del neoplatonismo islámico en el Liber de Causis. En este escrito, a pesar de haberse suprimido la instancia heneádica, se menciona la reditio completa de las sustancias intelectuales y no de la causa primera, la cual por ninguna razón debe justificarse que además de una deba ser trina.

En una muy definida intención de reapropiación de la tradición platónica en el sentido propuesto más arriba, Nicolás de Cusa escribe en 1459 -es decir, casi veinte años después de su célebre De docta ignorantia- el opúsculo De principio.

Este escrito cusano fue considerado por mucho tiempo un Sermón porque parte de un texto del Evangelio de Juan en el cual Cristo dice de sí mismo que él es el Principio y que "como tal" "nos" habla (Bormann 2002: XLIV-XLV). En este caso, el Cusano confronta explícitamente los dichos del evangelio de Juan con el procleano In Parmenidem y las alusiones a este texto, que son muchas, son perfectamente rastreables en la versión de Moerbeke. Con todo, no hay que desconsiderar el hecho de que, como dijimos, el Cusano cuenta por entonces con la versión de la Elementatio ya anotada. Incluso es probable que por este tiempo conozca cierto avance de la versión de De Platonis Theologia que realizaba Pedro Balbo y que será gravitante en una obra dos años posterior, De li non aliud.

Si se comparan ambas teologías procleanas, el término "authypóstaton" es utilizado con matices diferentes y, en este sentido, podemos decir que son mucho más complementarias la Elementatio e In Parmenidem que lo que presenta Proclo en la Theologia, donde el foco está puesto en la distinción entre 
los dioses y el modo de procesión, lo cual implica una modificación teórica en la henadología (Lankila 2010: 67).

Como sugiere Dodds en su todavía imprescindible comentario a la Elementatio, estas dos nociones que aparecen diferenciadas en ese texto se unifican en la presentación que realiza Proclo en De Platonis Theologia donde autoconstitución y autarquía son dos propiedades indiferenciadas del mundo espiritual de los dioses (Dodds 1963: 196 y 224).

El Cusano, aun cuando conozca muy bien la Theologia, no atenderá especialmente a todo este complejo mundo de dioses procleano, solo le bastarán ciertas alusiones críticas a su "politeísmo", único flanco débil de su admiradísimo pensador ateniense.

Particularmente en De Principio, las concordancias que señala entre su pensamiento en cuanto cristiano y el pensamiento de Proclo son permanentes a tal punto que un estudioso como Flasch ha sostenido, quizá un poco exageradamente, que pone en pie de igualdad las palabras de dos autoridades: Cristo y Proclo (Flasch 1998: 500).

Según el Cusano, Proclo ha sostenido con razones filosóficas lo que Cristo simplemente enunció, a saber: solo lo uno es necesario. Esto atiende no solo a la unidad sino a la unicidad del primer principio: si hubiera varios principios serían semejantes en que todos ellos participarían de lo uno. Así pues, no hay más que un ser necesario fuera del cual nada puede ser, como afirmaron Cristo y Parménides, en referencia al personaje del diálogo platónico (cfr. De princ., n. 6-8).

En una obra inmediatamente anterior, De Beryllo de 1458, el Cusano había evaluado la tradición filosófica a la luz de la noción clave de su propia filosofia: la coincidentia oppositorum. En esta evaluación ocupan el primer lugar los filósofos y teólogos de la tradición platónica: el propio Platón pero también Dionisio y Proclo. Precisamente la vía negativa inaugurada por Platón, según el Cusano, permite a los autores de esta tradición pensar más allá de la disyunción de opuestos. Esto no les está permitido a Aristóteles y a los peripatéticos en general quienes están sujetos al principio de no contradicción.

Este es un tema muy importante para la filosofia cusana. Desde su escrito De docta ignorantia donde postula la coincidentia oppositorum ha criticado duramente a Aristóteles. Como señala Steel (2003: 582-585), se puede establecer muy claramente una relación entre la crítica que aparece en el libro VII del In Parmenidem de Proclo con la crítica del Cusano:

Et michi videtur et non admittens post ipsum (sc. Aristoteles) unum quod supra intellectum, eo quod contradictionis axiomate omnino persuasus; videns autem indicibile illud et ineloquibile apparens, stare usque ad intellectualem causam et intellectum, supra... totorum causam (In Parm. VII KL 72 1.5-9). 
Ahora bien, podríamos preguntarnos si pensar un principio-uno que esté más allá de los opuestos implica también para el Cusano pensar que tal principio es supraintelectual.

Para responder a esta pregunta el concepto de authypóstaton se vuelve central.

Toda la argumentación cusana en De principio reside en mostrar que todo lo divisible procede de una causa indivisible. Lo que se divide, puesto que puede no ser, no subsiste por sí. Por lo tanto, solo lo indivisible es authypóstaton, es decir per se subsistens. El Cusano utiliza el término en griego y solo en el comienzo lo traduce por su equivalente latino, en el resto de la obra mantendrá su peculiar escritura griega (Honecker 1938):

Primum igitur investigemus, si est principium. Plato, prout Proclus in commentariis Parmenidis scribit, asseruit hunc mundum ex seniori causa in esse prodiisse, nam partibile non potest per se subsistere; quod enim per se subsistit, hoc est quod esse potest. Partibile autem, cum possit partiri, potest non esse. Undecum, quantum est de se, possit partiri et non esse, patet quod non est per se subsistens sive authypóstaton. (De princ., n.2).

El Cusano reúne indivisibilidad y authypóstaton, esto significa que para él la autoconstitución no supone duplicidad. Esto mismo es para Proclo: las hénadas son prioritariamente authypóstata porque su movimiento de procedencia y retorno no implica alteridad. Esto explica que sean una instancia prenoética: las hénadas no son pensamiento del pensamiento ( $\mathrm{ffr}$. In Parm.VII Co. 1150 1.25-30).

El Cusano también afirma la no alteridad del authypóstaton: en el subsistente por sí son lo mismo el que hace y lo hecho, el generante y lo generado (cfr. De princ., n.3). Estas expresiones que evocan un lenguaje tradicional relativo al tema trinitario, es ligado por el Cusano en varios pasajes de su texto con otra noción procleana: auto-hén, mencionada en latín como auto-unum (cfr. De princ., n. 8 y n. 26).

En In Parmenidem aparecen como equivalentes "auto-unum", "primum idem" " "primus deus", "autodeus", "autodeitas" (cfr. In Parm,VI Co 1096 1. 17-19). $\mathrm{Al}$ igual que en la Elementatio, todo dios es participable excepto lo uno ( $\mathrm{f} f \mathrm{r}$. Prop. 116). El Cusano atendiendo a esta lectura procleana de Platón afirma:

Et ideo haec imitas, quae unum absolutum per Platonem nominator [...] Ille est ipsa unitas, quae et autounum, per se scilicet unum, licet melius sit omni nominabili et authypostato, ut infra dicetur (De princ., n. 8). 
Una clave para entender la posición cusana es la consideración de distintas perspectivas en su abordaje. Según esas distintas perspectivas, el authypóstaton es el primer principio intelectual -como en otros autores cristianos- y su autoconstitución es equiparable al pensamiento del pensamiento; o bien el authypóstaton es supraintelectual y de allí se infiere que ni siquiera puede ser llamado con propiedad con este nombre.

Así pues, el Cusano fluctúa en la consideración del principio como principio intelectual o principio más allá del intelecto. Cuando lo entiende como intelectual equipara authypóstaton y Trinidad. El Principio engendra de sí mismo su razón o lógos. En esta "definición de sí” se conoce a sí mismo y a todo:

Et ideo rationem sui seu diffinitionem seu logon de se generât. Quae diffinitio est ratio, in qua se unum necessarium intelligit et omnia, quae unitate constringuntur et fieri possunt. Et logon est consubstantiale verbum seu ratio difiniti patris se diffinientis in se omne diffinibile complicans, cum nihil sine ratione unius necessarii diffiniri possit (De princ. n.9).

El lógos es lo definido que se define a sí mismo complicando en sí todo lo definible. Debemos advertir el sentido del término "diffinire" que significa poner límite (finis), en este contexto se trata del límite ontológico, es decir ser causa del ente determinado: nada puede ser definido sin la razón o lógos del uno necesario. Así el principio se constituye en una dialéctica por la cual al principio en la eternidad (Padre) le corresponde un principiado en la eternidad, principio principiado eterno (Hijo) y el principiado de ambos (Espíritu).A lo cual agrega el Cusano la concordancia con el platonismo: esta Trinidad es única esencia de la eternidad a la que Platón llamó "uno" (cfr. De princ., n.10-11).

El tono filosófico con el cual el Cusano trata la Trinidad se matiza cuando advierte el auténtico escándalo filosófico que constituye postular un "principio del principio". Ningún filósofo admitiría esta regresión al infinito:

Diceres: turbat audientem, quando dicis principii esse principium; hoc enim nullus philosophorum admittit, ne procedatur sic in infi nitum et sublata sit omnis veritatis inquisitio, quando ad primum principium pertingi non posset (De princ., n. 13).

Evidentemente, cuando dice "ningún filósofo" debería agregar "aristotélico o peripatético", pues a continuación admite que esto se soluciona pensando que en lo infinito eterno en acto hay coincidencia entre principio y principiado, es decir, superando el principio de no contradicción 
y pensando la eternidad como un infinito en acto ( $f f r$. De princ. 13). Con todo, las objeciones no son exclusivamente para los aristotélicos sino que los platónicos también tienen su parte. Ellos se equivocan, dice el Cusano, al considerar que la eternidad supone alteridad, esto es al suponer una multitud en la eternidad como pueden serlo una pluralidad de hénadas o dioses ( $c f r$. De princ., n.17).

Cuando el authypóstaton es comprendido como trinidad, los movimientos de procesión y retorno son concebidos como el engendramiento eterno y, al mismo tiempo, como pensamiento del pensamiento sin alteridad. Sin embargo, para el Cusano la imagen del intelecto que se piensa a sí mismo es solo un "enigma" o ejemplo simbólico de lo que es incomprensible. Dicho en los términos paradojales que el Cusano suele usar:"Et non possumus negare, quin se intelligat, cum melius sit se inteligente" (De princ. n.9) frase en la cual el "melius" tiene el sentido de "supra": se intelige a sí mismo aunque está por sobre lo que se intelige.

En esta consideración del principio como supraintelectual el Cusano pone en duda también la pertinencia del término "authypóstaton" y prefiere considerarlo en los términos de la via negativa. "Authypóstaton" resulta un "vocablo conveniente" toda vez que se entienda por eso "lo que no es por otro". La autosubsistencia se reconvierte con el vocabulario que anuncia la noción cusana de "non aliud" tanto en su dimensión ontológica como gnoseológico-predicativa. Lo que no es por otro es no otro y su no otredad lo define tanto en la determinación de lo que es cuanto en su posibilidad de ser conocido y nombrado:

Principium enim, cum non sit ab alio, per se subsistere dicimus, cum nihil esse concipere valeamus, si ipsum non conciperemus esse; primum enim, quod se offert conceptui, est ens, deinde ens tale; et licet principium entis nihil entium sit, cum principium nihil sit principiad, tamen, nisi concipiamus principium esse, nullum de ipso formare possumus conceptum (De princ. n.18).

A continuación, el Cusano vuelve a afirmar que Platón vio esto y advirtió que si se quita de lo uno todo lo pasivo, lo contracto, es decir todo límite y toda determinación, se alcanza lo uno en sí mismo y desligado de todo. Y al verlo de esta manera Platón no pudo sino negar todo de tal uno absoluto: negarle el ser, pero también negarle el no ser, negarle la subsistencia por sí -esto es su carácter de authypóstaton- y hasta negarle el hecho de que sea principio y que sea uno. En un pasaje que representa una formulación hiperbólica de la vía negativa, el Cusano niega todo opuesto respecto de lo uno con las locuciones "nec... nec..." y niega que pueda predicarse con propiedad cualquier proposición con cópula "est” en tanto 
que el principio de todo lo nominable debe ser, él mismo, innominable:

Et ut sic videtur, nec est ens nec non ens nec est nec subsistit nec est subsistens nec per se subsistens nec principium, immo nec unum. Immo non esset apta locutio 'unum est unum', cum copula illa est non possit uni convenire, nec sine copula dicendo sic 'unum unum' esset apta locutio, cum omnis locutio, quae sine alteritate aut dualitate non est proferibilis, non conveniat uni. Unde si attendis, tunc principium omnium nominabilium, cum nihil possit principiatorum esse, est innominabile, et ideo etiam non nominari principium, sed esse principii nominabilis innominabile principium omne qualitercumque nominabile antecedens sicut melius (De princ., n. 19).

En lo que sigue, el Cusano muestra la inconveniencia de todo vocabulario afirmativo para referirse a lo absoluto. La senda del lenguaje negativo le permite retomar el comentario procleano con toda su fuerza. Lo "uno-uno" sin cópula es más bien lo no uno que antecede a todo lo que es uno y este es el que llamamos impropiamente, authypóstaton. Como anticipamos, Proclo mismo había declarado la inconveniencia de predicarlo del principio primero aunque debemos agregar ahora que en In Parmenidem aclara que solo podría hacerse cuando lo nombramos en cuanto causa de los authypóstata, es decir de las hénadas. A este primero, que es lo uno, lo llama "dios de dioses" dándole todas las notas negativas, pues, como advierte el Cusano, Proclo se dio cuenta de que lo uno está más allá y es anterior a la disyunción entre afirmación y negación (cfr. De Princ., n.24). Sin embargo, Proclo se equivoca al sostener que pueda haber muchos coeternos con el uno, excepto sus tres hipóstasis, porque la eternidad no puede multiplicarse, lo uno es indivisible e inmultiplicable ( $f$ r. De Princ., n.25). Hay dos notas que señalar. Primero la ya mencionada crítica al politeísmo procleano. En segundo lugar el hecho de que para el Cusano las tres hipóstasis platónicas -para nosotros "neoplatónicas"- uno, intelecto y alma, no suponen alteridad y son perfectamente asimilables a la trinidad cristiana, tal como lo había considerado Agustín en De civitate dei y antes de él Mario Victorino en sus textos contra los arrianos.

Para el Cusano, el "error" de Proclo es reparado por su propio maestro, Platón, quien no multiplicó los "subsistentes por sí". En varias ocasiones, el Cusano abandona el comentario para dirigirse al propio texto platónico, Parménides, del cual poseía una nueva versión completa encargada por él mismo a Jorge de Trebisonda (Ruocco 2003). Es necesario recordar que el texto del diálogo platónico ofrecido por Moerbeke junto al comentario llega solo hasta el fin de la primera hipótesis. El "elogio de Platón" concierne fundamentalmente a que él es quien entendió no solo que de lo uno 
debe ser negado todo, sino que la mónada es principio del número, que la unidad es siempre anterior y que la multiplicidad (que no puede ser eterna) no puede estar separada de lo uno. Así, solo hay uno en dos sentidos: uno en sí o imparticipable, lo uno en otro o participable (cfr. De princ. n.28-30). Como se ve, el Cusano pasa del elogio a Platón a la terminología procleana que alude a la participabilidad y la imparticipabilidad: tal uno que Platón pensó como más allá de todo es imparticipable cuando es considerado en sí mismo y participable cuando es considerado desde el punto de vista de su comunicación en lo múltiple. De este modo, al ascender al principio, las oposiciones del mundo se piensan como una pues el principio complica en sí tanto lo que es cuanto lo que no es y la multiplicidad dada no es sino mostración o participación en la alteridad del principio imparticipable. En términos cusanos:

Dum video per contradictoria principium, omnia in ipso video; esse enim et non esse omnia ambit, quondam omne, quod did aut cogitari potest, aut est aut non est. Principium igitur, quod est ante contradictionem, omnia complicat, quae contradictio ambit. [...] Omnis igitur creatura imparticipabile principium in alteritate participat, sicut aequalitas imparticipabilis < participatur> in similitudine (De princ. n. 36).

Por último, podemos preguntarnos cuáles son las implicancias epistemológicas de la consideración de un principio como intelectual o como supraintelectual, dicho de otro modo cuál es la posibilidad de acceso a tal principio.

En esta cuestión, el Cusano hace referencia a una doctrina típicamente procleana. Proclo decía que no solo la naturaleza intelectual accede a Dios pues también la naturaleza no intelectual aspira a lo uno: si todo por lo uno es lo que es, entonces todo sin excepción lo desea en tanto lo uno penetra todo lo que es. Tal doctrina es reapropiada por él en términos cristianos: la creatura por sí misma es nada y tiene todo el ser de su causa. Todo, sin excepción, encuentra su ser verdadero en su principio o Lógos. Es necesario, pues, que el principio supraintelectual se revista de la naturaleza intelectual y aun de la naturaleza sensible para que pueda ser alcanzado de algún modo. He aquí una clara referencia a la encarnación del Lógos. El ejemplo elegido por el Cusano, como "enigma" de esta relación inaprehensible es el de la palabra humana y el verbo mental: cuando el intelecto quiere manifestar el verbo mental de sí mismo, en el cual se entiende, lo hace por medio de la palabra o la escritura o de otra designación sensible. Así, el verbo, antes de la designación es mental, pero proferido se reviste de la especie sensible, y así lo no sensible se constituye como sensible (cfr. De Princ, n.37-38). 
El Principio no es lo mismo ni lo diferente respecto de las creaturas pues está más allá de la identidad y la alteridad, pero se hace visible:"El Principio nos habla", como dice el texto que abre la reflexión cusana, es decir se vuelve Lógos, Palabra intelectual eterna, y se vuelve Cristo, Palabra sensible y encarnada.

De este modo, el Cusano, lector directo de Proclo, conocedor además de una tradición de pensadores anteriores a él quienes asimilaron algunas de las doctrinas a su propio pensamiento o lo pusieron en concordancia con otras tradiciones, dio un paso decisivo en la lectura filosófica de los misterios del cristianismo con las herramientas conceptuales del diádoco de Platón: la noción de authypóstaton, la radicalidad de la vía negativa y el más grande de los misterios del cristianismo comprendido, tal como lo había hecho el Areopagita sin declarar sus fuentes, como manifestación de lo escondido o bien participabilidad de lo imparticipable.

\section{BIBLIOGRAFIA}

Beierwaltes, W. (1987), "Das Seiende Eine. Zur neuplatonischen Interpretation der zweiten Hypothesis des platonischen Parmenides: das Beispiel Cusanus”, en: G. Boss - G. Steel (eds.), Proclus et son influence (Actes du colloque de Neuchatel, Juin 1985), Zurich: 287-297.

Bertholdus de Mosburch (2001), Expositio super Elementationem theologicam Procli. Propositiones 35-65, ed. A. Sannino (Hamburg: Felix MeinerVerlag).

Bormann, K. (1986) (ed.), Cusanus-Texte III Marginalien 2. Proclus latinus 2.2 Expositio in Parmenidem Platonis (Heidelberg: C. Winter).

Bormann, K. (2001), "Affirmation und Negation, der Parmenides-Kommentar des Proklos in Nikolaus von Kues' Schrift 'Tu quis es”, Theologische Quatalschrift, 181: 84-96.

Bormann, K. (2002), "Einleitung”, en Nikolaus von Kues, Philosophische-theologische Werke, Band 1 (Hamburg: Felix Meiner Verlag).

Calma, D. (2019), Reading Proclus and the Book of Causes, vol. 1 (Leiden: Brill).

D’Amico, C. (2009), "La recepción del pensamiento de Proclo en la obra de Nicolás de Cusa", Anales del Seminario de Historia de la Filosofía, 26: 107-134.

D’Amico, C. (2011), "Proclus" en Encyclopédie des Mystiques Rhénans. D”Eckhart à Nicolas de Cues et leur réception, Édition française par Marie-Anne Vannier, Préface Bernard McGinn (Paris: Les Éditions du Cerf, 1003-1009).

Dodds, E. R. (1963), Proclus. The Elements of Theology. A Revised Text with Translation, Introduction and Commentary (Oxford: Clarendon Press).

Flasch, K. (1984), Einleitung, in Berthold von Moosburg, Expositio I: XI-XXXVIII (Hamburg: Felix Meiner). 
Flasch, K. (1998), Nikolaus von Kues. Geschichte einer Entwicklung. Vorlesungen zur Einführung in seine Philosophie (Frankfurt am Main:Vittorio Klostermann).

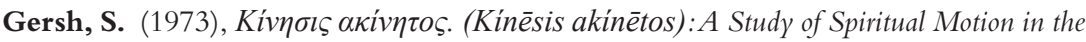
Philosophy of Proclus (Leiden: Brill).

Gersh, S. (2014) (ed.), Interpreting Proclus: From Antiquity to the Renaissance, (Cambridge: Cambridge University Press).

Gersh, S. (2017). "Proclus in the History of Philosophy: Construction and Deconstruction”, en D. Layne y D. D. Butorac (eds.), Proclus and his Legacy (Berlín: Walter de Gruyter, vol. 65, 17-32)

Guildentops, G. (2019), "Bate et sa lecture 'encyclopédiste' de Proclus”, en D. Calma, Reading Proclus and the Book of Causes (Leiden: Brill, vol. 1, 352-375).

Henricus Bate (1990), Speculum divinorum et quorundam naturalium. Parts XI-XII: On Platonic Philosophy, edited by. H. Boese with an introduction and an analysis by C. Steel (Leuven: Leuven University Press).

Honecker, M. (1938), "Nikolaus von Cues und die griechische Sprache", Sitzungsberichte der Heidelberger Akademie der Wissenschaften, Philosophisch-Historische Klasse (1937/38, 2. Abhandlung) (Heidelberg Akademie: Heidelberg).

Imbach, R. (1978), “Le (néo-)platonisme medieval, Proclus latin et l'école dominicaine allemande", Revue de Théologie et de Philosophie, 110: 427-448.

Klibansky, R. (1929), Ein Proklos-Fund und seine Bedeutung (Heidelberg: C. Winter).

Lankila, T. (2010), "Henadology in the two theologies of Proclus", Dionysius, 28: 63-76.

Ludueña, E. (2016), "Dioses, inteligencias y ángeles de Alberto Magno a Bertoldo de Moosburg”, Cuadernos de Filosofía, 66: 47-59.

Nicolaus Cusanus (2007), Apologia doctae ignorantiae, Opera omnia. Volumen II, ed. R. Klibansky. Editio stereotype W. Beierwaltes - H.-G. Senger (Hamburg: Felix Meiner).

Nicolaus Cusanus (1988), De deo unitrino principio, Tu quis es (De principio). Opera Omnia. Volumen X. Opuscula II. Fasciculus 2 b, edited by K. Bormann and A. D. Riemann (Hamburg: Felix Meiner).

Proclus (1987), Elementatio Theologica, translata a Guillielmo de Moerbecca, ed. H. Boese (Louvain: Louvain University Press).

Proclus (1982), Commentaire sur le Parménide de Platon. Traduction de Guillaume de Moerbeke. Tome I: Livres I à IV,édition critique par C. Steel (Leuven - Leiden: Leuven University Press- Brill).

Proclus (1985), Commentaire sur le Parménide de Platon. Traduction de Guillaume de Moerbeke. Tome II: Livres V à VII et Notes marginales de Nicolas de Cues, édition critique par C. Steel, suivi de l'édition des extracts du Commentaire sur le Timée, traduits par Moerbeke (Leuven - Leiden: Leuven University Press - Brill).

Ruocco, I. (2003), Il Platone latino. Il Parmenide: Giorgio di Trebisonda e il cardinale Cusano, (Firenze: Leo S. Olschki Editore).

Secchi, P. (2018), "Cusano e Proclo: Le mediazioni platoniche” en Historia philosophica. An International Journal, 16: 19-45. 
Senger, H.-G. (1986), Cusanus-Texte III Marginalien 2. Proclus latinus. 2.1. Theologia Platonis, Elementatio theologica (Heidelberg: C. Winter).

Steel, C. (1990), "Introduction", en Henricus Bate, Speculum divinorum et quorundam naturalium. Parts XI-XII: On Platonic Philosophy, edited by H. Boese, IX-XXV (Leuven: Leuven University Press).

Steel, C. (2003). "Beyond the principle of contradiction? Proclus' Parmenides and the origin of negative theology",Miscelanea Mediaevalia 30: 581-599.

Steel, C. (2014), "William of Moerbeke, translator of Proclus" en S. Gersh (ed.), Interpreting Proclus. From Antiquity to the Renaissance, (Cambridge: Cambridge University Press, 247-263).

Sturlese, L. (1987), “Il dibattito sul Proclo latino nel Medioevo fra l’Università di Parigi et lo Studium di Colonia”, dans G. Boss, G. Seel (éds.), Proclus et son influence, (Zürich: Grand Midi, 261-285).

van de Vyver, E. (1956), "Marginalia van Nicolaus van Cusa in Bate-Codex 271 en andere códices van de Koninklijke Bibliotheek te Brussel”, Tijdschrift voor Philosophie 18, (3): 439-456.

Recibido: 01-06-2020; aceptado: 03-07-2020 